\title{
Fractional derivative resolution of the anomalous magnetic field diffusion through a ferromagnetic steel rod: Application to eddy current testing.
}

\author{
B. Ducharne ${ }^{1,2}$, B. Zhang ${ }^{3}$, G. Sebald ${ }^{2}$ \\ ${ }^{1}$ Laboratoire de Génie Electrique et Ferroélectricité - INSA de Lyon, Villeurbanne, France. \\ 2 ELyTMaX UMI 3757, CNRS - Université de Lyon - Tohoku University, International Joint Unit, \\ Tohoku University, Sendai, Japan. \\ ${ }^{3}$ School of Mechanical, Electrical and Information Engineering, Shandong University, Weihai, China.
}




\section{Abstract:}

Electromagnetic evaluations are performed daily by steel manufacturers and steel user companies. Among different methods, Eddy Current Testing (ECT) is routinely used. It is versatile, simple, and conveys rich information. ECT evaluations on steel specimens remain complex to interpret quantitatively. Most steels are ferromagnetic and exhibit high nonlinear magnetic behaviors. Such nonlinearities are an issue when it comes to signal analysis and interpretation. The magnetic field diffusion in a steel rod is highly frequency-dependent. It is the direct consequence of the Eddy current generation. If the rod is ferromagnetic, the unstable distribution of the magnetic domains interacts with the macroscopic Eddy current circulation and perturbs the magnetic field diffusion, which becomes anomalous. Fractional derivative diffusion equations are appropriate tools for the simulation of these phenomena. The fractional order acts as an adjustment parameter that provides flexibility in the simulation method. In this study, a 2D fractional diffusion equation has been solved by using finite differences in polar coordinates to anticipate the magnetic field diffusion and the ECT measurement in a steel rod specimen. Defects have been simulated through local variations of the physical properties. Good simulation results have been obtained and validate the fractional diffusion equation as an efficient method for the simulation of the anomalous diffusion magnetic field and the interpretation of ECT measurements.

\section{Keywords:}

Fractional derivative, anomalous magnetic diffusion, steel rod, eddy current testing 


\section{1 - Introduction:}

Eddy Current Testing (ECT) is one of many electromagnetic testing methods used in Nondestructive Testing (NDT). ECT uses electromagnetic induction to detect and characterize surface and sub-surface flaws in conductive materials. The first ECT developments and reports were proposed at the beginning of the $20^{\text {th }}$ century. What's made ECT so widespread can be explained in its simplicity, its ability to work on every electrically conductive material, and its versatility [1]-[3]. The panel of ECT applications is large and includes surface crack detection [4], material sorting [5], thickness assessment [6], and tube testing [7]. For non-ferromagnetic materials such as aluminum and copper, ECT can be used to determine metal purity [8]. It is also used to evaluate the fibers' uniformity, and alignment in carbon fiber reinforced polymers [9].

ECT is attractive for many reasons, such as low cost, fast answer, little space, and maintenance. However, for ferromagnetic materials characterized by intense magnetic responses, ECT scopes and performances have to be considered differently as the linear behavior observed for nonferrous materials becomes strongly nonlinear. ECT devices provide electrical signals; for efficient use of these devices, a correct interpretation and understanding of this electrical information are mandatory. For many years now, industrials have shown interest in developing simulation tools reducing the experimental campaigns and improving their products' knowledge and performances.

In steel companies, ECT can be used at different stages of the manufacturing process. In this study, we mainly focused on the surface scan of rod-shaped steel specimens. Various ECT methods can be envisaged for the evaluation of these rods: 
_ The real-time monitoring of a wound coil impedance. Fig. 1 below gives an illustration of this method. The rod-shaped specimen is moving in a horizontal direction. A defect manifests itself as a spontaneous variation of the sensor impedance [1][2].

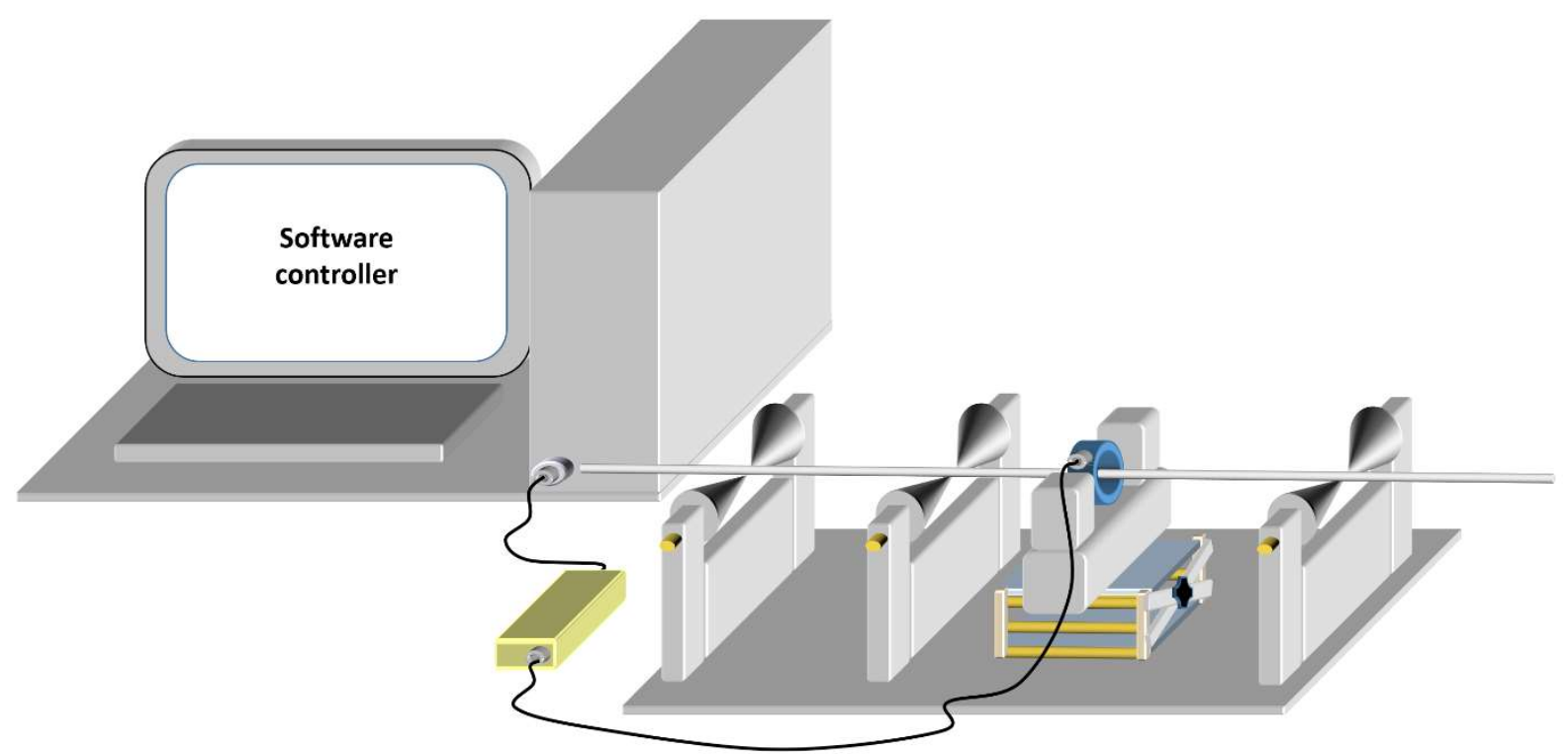

Fig. 1 - Overall 3D view of the industrial setup used to evaluate a rod-shaped steel specimen with an ECT single-coil method.

_ The real-time monitoring of a Transmitter/Receiver sensor (TR probe). The electromagnetic working principles of the TR probe can be assimilated to those of a single-phase transformer. The transmitter coil connected to an alternative voltage source generates an alternating magnetic field which magnetizes the ferromagnetic rod. This magnetization induces a flux variation and an electromotive force in the receiver coil. The tested specimen is translating inside the TR probe, and a defect is detected through a sudden variation of the receiver electromotive force [10][11].

_ A differential method: two similar and juxtaposed coils are required. The experimental conditions are close to those of Fig. 1. The rod-shaped specimen is magnetized first (from a permanent magnet or the electrical current ( $A C$ or $D C$ ) passing through a wounded additional coil). It goes incrementally through the first and the second coil. The real-time differential 
voltage of these two coils is monitored; a defect manifests itself by a brief variation of this voltage [12].

The magnetization behavior is a material characteristic. It depends on the excitation waveform (frequency, amplitude), on the geometry, and the nature of the tested specimen. As magnetization for ferromagnetic materials is very sensitive, minor variations can generate significant variations of the sensor measurements.

Careful considerations of the magnetic properties are mandatory for an efficient simulation of the ECT device behavior. Classic methods combine a nonlinear, hysteretic material law to the Maxwell equation solved with a space discretized method (finite elements, finite differences) [13]-[16]. These methods are popular, but accurate simulation results can only be obtained through the consideration of a frequency-dependent material law complex to simulate. Once combined with the space discretized method, it most of the time ends up in oversized resolution times or divergent results.

In [17][18], the authors proposed an alternative method using fractional derivative operators. The magnetic field diffusion in a ferromagnetic component interacts with local magnetic behaviors, including the magnetic domain kinetics. These interactions disturb the diffusion process, which becomes anomalous. An anomalous diffusion equation was obtained by replacing the first-order time derivation by a fractional-order derivative in the classic magnetic diffusion equation:

$$
\nabla^{2} \vec{H}=\sigma \frac{d \vec{B}}{d t} \rightarrow \nabla^{2} \vec{H}=\sigma^{\prime} \frac{d^{\alpha} \vec{B}}{d t^{\alpha}}
$$

The simulation method described in [17][18] is promising. However, in [17], the study was restricted to magnetic laminations and the spatial resolution of the anomalous diffusion equation to one dimension. Quite similarly, in [18], the study was limited to the rectangular 
shape cross-section of a massive toroidal core. A 2D discretization in a Cartesian coordinates system was enough to reach convergence and accurate simulation results.

In this new study, the objective was to simulate the ferromagnetic behavior of rod-shaped steel specimens. We opted for a 2D resolution in a polar coordinate system:

$$
\nabla^{2} \vec{H}=\sigma \frac{d \vec{B}}{d t} \rightarrow \frac{\partial^{2} \vec{H}}{\partial r^{2}}+\frac{1}{r} \frac{\partial \vec{H}}{\partial r}+\frac{1}{r^{2}} \frac{\partial \vec{H}}{\partial \theta^{2}}=\sigma^{\prime} \frac{d^{\alpha} \vec{B}}{d t^{\alpha}}
$$

Finite differences have been used to space discretized the cross-section of tested specimens, and the local properties have been modified to take into account the influence of natural defects. Eventually, the Maxwell equations (Faraday Maxwell equation, Gauss's law) were used to establish the link between the magnetic and the electrical quantities.

The structure of this manuscript can be summarized as follows:

Section 2 describes the experimental setup and the tested specimens. The simulation method is described in detail in section 3 , including the defects taken into account and some additional explanations about fractional derivatives and their physical meaning in the context of the anomalous magnetic field diffusion. The first simulation results are provided and commented in this section, such as a refined analysis of the simulation parameters influence (electrical conductivity, fractional derivative order). Section 4 provides a comparison of experimental measurement/simulation in an ECT situation. The good accuracy observed is worth a validation of the simulation method. Conclusions and perspectives are provided in the last section.

\section{2 - Experimental setup}

\section{a) Experimental setup description}


A dedicated experimental setup has been designed for the validation of our simulation results. The same magnetic characterization sensor (Fig. 2) has been used successively to plot the tested specimen hysteresis cycles and measure their ECT answers. Concerning the hysteresis cycle characterization, a frequency generator Agilent 32220A was used to drive a power amplifier Kepco BOP 100-4M in a regulated current configuration. The Kepco amplifier was the energy source. It supplied both the two 500 turns coils visible on both the extremities of the characterization sensor. These excitation coils were plugged in series. They acted as micro Helmholtz coils, and they were explicitly designed to generate a magnetic field excitation confined in the rod-shaped specimens and collinear to the $\overrightarrow{O_{x}}$ direction (Fig. 2). As illustrated below (Fig. 8), on the detailed illustration of the characterization sensor, two symmetrical sensor coils, 60 turns each, were used for the magnetic flux monitoring (plugged in series).

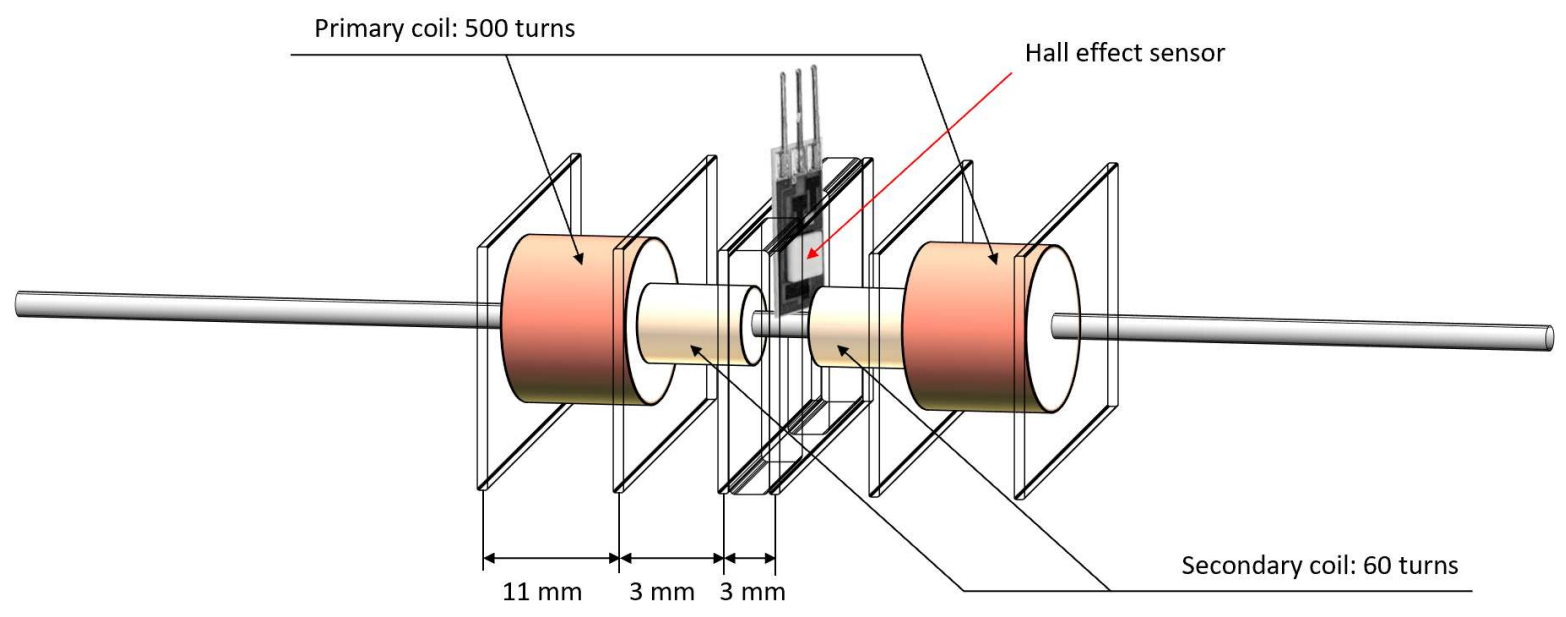

Fig. 2 - Detailed description of the magnetic characterization sensor.

A noise shielded radiometric linear Hall probe (SS94A from Honeywell) was positioned tangent to the tested sample, just in the middle of the sensor coils. The data acquisition of both the sensor coils electromotive force and of the Hall effect sensor were ensured by a DEWESoftX2 data acquisition software associated with a SIRIUS 8×CAN data acquisition 
system. The numerical integration and the drift correction of the sensor coils signal were performed in a post-processing stage with Matlab ${ }^{\mathrm{TM}}$ software. The hysteresis cycles were also plotted using Matlab ${ }^{\mathrm{TM}}$ software.

An overall 3D view of the ECT experimental setup is depicted in Fig. 3 below:

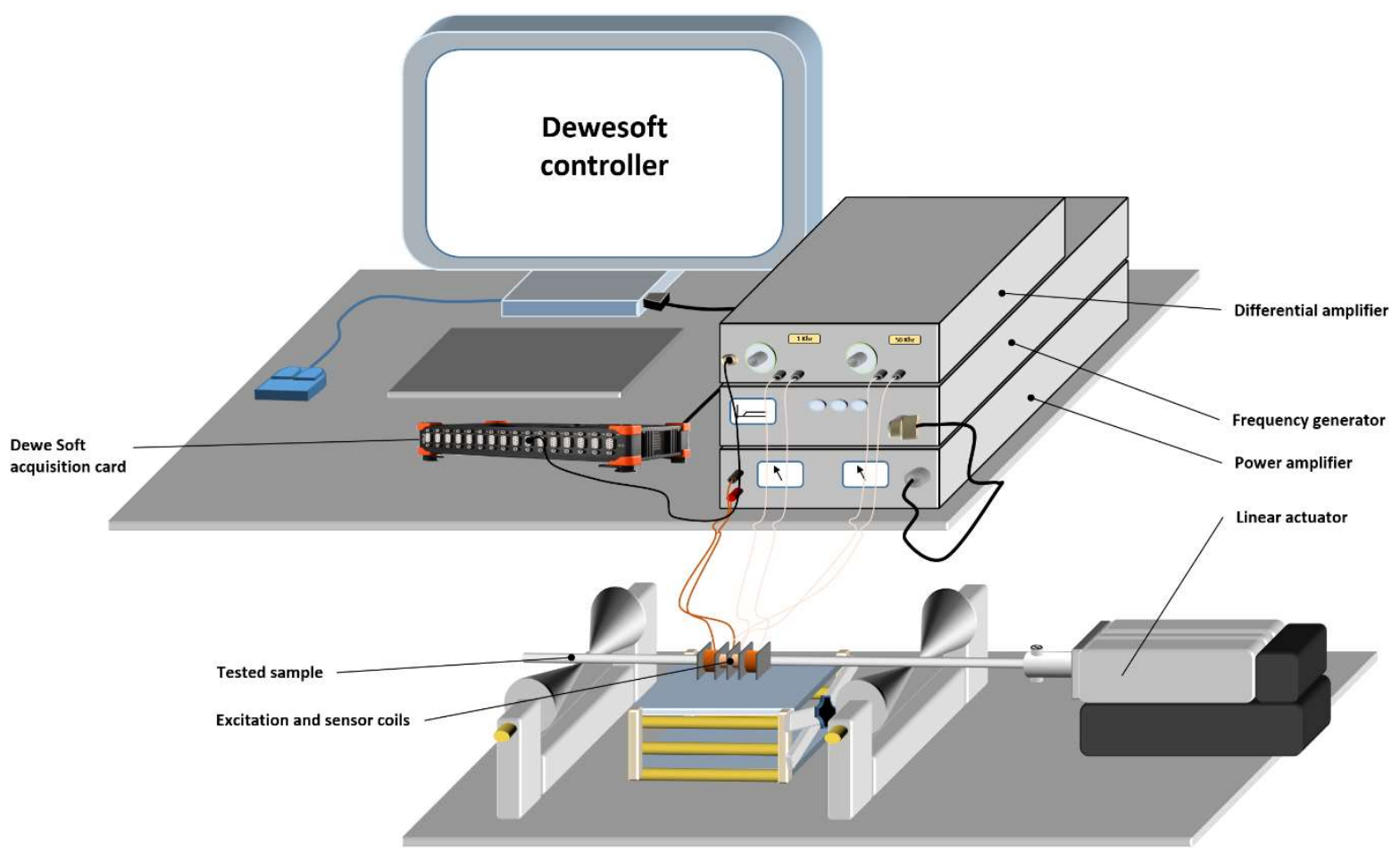

Fig. 3 - Overall 3D view of the experimental characterization setup.

The magnetic field excitation was generated the same way as we did for the hysteresis cycle characterization. Tangent surface $\vec{H}$ frequency and amplitude were set to $500 \mathrm{~Hz}$ and 1 $k A \cdot m^{-1}$, respectively. We intentionally worked with high amplitude magnetic excitations to validate the viability of the simulation method even under a well-defined nonlinear environment. Translation movements of the tested specimen were ensured from a SKF, MAX10 linear actuator at a speed of $8 \mathrm{~mm} \cdot \mathrm{s}^{-1}$. The 60 turns coils were disconnected and monitored separately. The differential measurement of these two coils was filtered and amplified through a dual-channel filter, Stanford research systems model SR650 (in a $3 \mathrm{kHz}$, low-pass configuration). The DEWESoftX2 data acquisition software associated with the 
SIRIUSif $8 \times$ CAN data acquisition system was used again for the signals acquisitions. The RMS value of the resulting signal was calculated in a post-processing step using Matlab ${ }^{\mathrm{TM}}$ software. $^{-}$

\section{b) Tested specimen}

All the tested specimens were high carbon steel $(\approx 0.7 \%)$, of $1.5 \mathrm{~mm}$ radius. The electrical conductivity of these samples measured at room temperature is $510^{6} \mathrm{~S} . \mathrm{m}^{-1}$. These specimens are particularly strong, and can be used for springs, edged tools, and high-strength wires. The composition of the tested samples is depicted in Tab. 1 below:

Tab. 1 - High carbon steel specimens composition.

Composition (\%)

\begin{tabular}{c|c|c|c|c|c}
$\mathbf{C}$ & $\mathbf{M n}$ & $\mathbf{S i}$ & $\mathbf{C r}$ & $\mathbf{N i}$ & $\mathbf{P}, \mathbf{S}, \mathbf{C u}, \mathbf{N}, \mathbf{A l}, \mathbf{M o}$ \\
\hline 0.7 & 0.495 & 0.2 & 0.02 & 0.015 & trace amount
\end{tabular}

To test and validate our simulation method under the presence of a defect, a hole of $500 \times 300 \mu \mathrm{m}$ diameter and depth respectively has been intentionally drilled on the surface of two tested specimens (see Fig. 4).

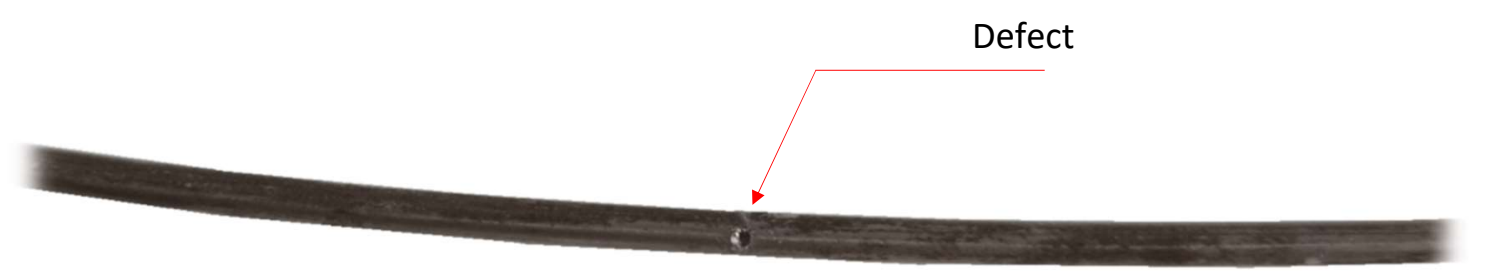

Fig. 4 - Tested specimen, including an additional defect (hole of $500 \mu \mathrm{m}$ diameter, $300 \mu \mathrm{m}$ depth).

\section{3-Simulation method}

Fig. 5 below shows a tested specimen and the experimental sensor specially designed to characterize the magnetic behavior and described previously in section 2 "experimental setup". 


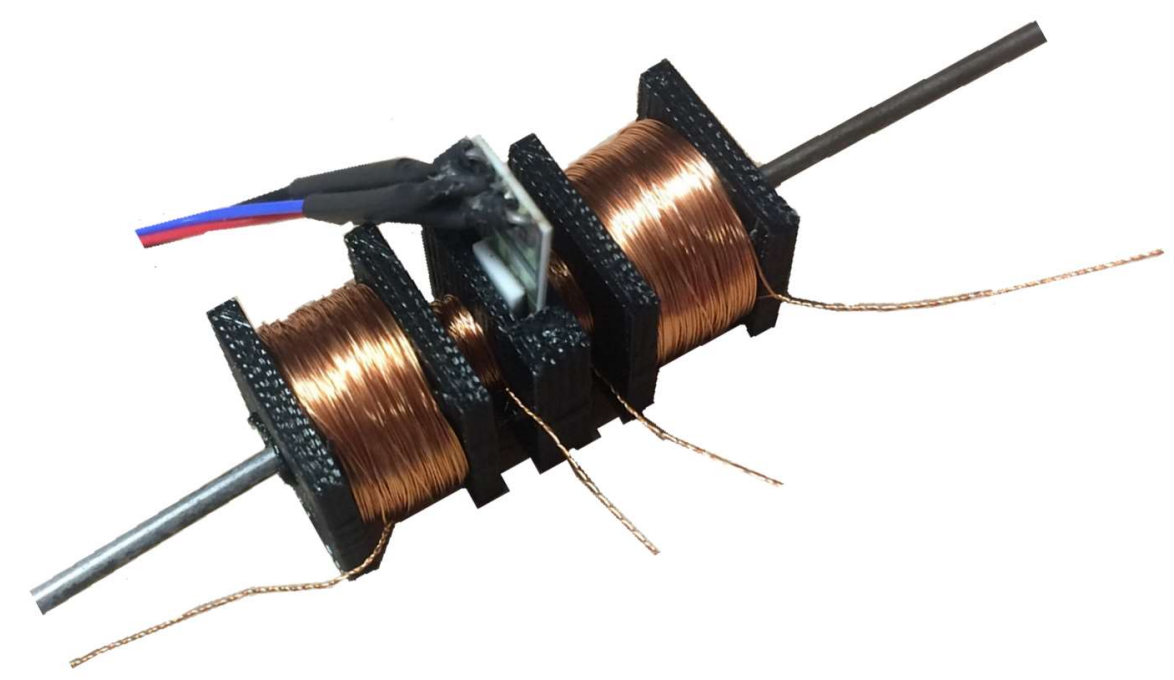

Fig. 5 - Picture of a rod-shaped specimen and the magnetic characterization sensor.

Even if vector quantities take place in the fractional magnetic diffusion equation (Eq.1), the first step of the simulation process consists in the reduction of this equation to scalar quantities, considering that $\vec{H}$ and $\vec{B}$ have only non-zero components along the z-axis.

$$
\nabla^{2} \vec{H}=\sigma^{\prime} \frac{d^{\alpha} \vec{B}}{d t^{\alpha}} \rightarrow \nabla^{2} H=\sigma^{\prime} \frac{d^{\alpha} B}{d t^{\alpha}}
$$

This simplification is possible under the following conditions:

_ A large ratio between the cylinder length and radius.

_ A large ratio between the excitation and the sensor coil widths.

_ A symmetrical distribution of the excitation and sensor coils along the z-axis.

_ A limited width of the sensor coils.

All those geometrical assumptions were respected by the developed sensor (see Fig. 2). $\vec{B}$ and $\vec{H}$ were therefore considered collinear in the scanned area and were replaced by their magnitudes in the updated version of Eq. 1. Finally, the simulation method was limited to a 2D space discretization of the cylinder cross-section.

a) The numerical resolution of the $2 \mathrm{D}$ anomalous magnetic field diffusion equation in a polar coordinate system. 
Finite differences were used for the discretization of the anomalous diffusion equation spatial term. Since the rod specimen cross-section was a disc, we opted for polar coordinates.

$$
\nabla^{2} H=\frac{\partial^{2} H(r, \theta, t)}{\partial r^{2}}+\frac{1}{r} \frac{\partial H(r, \theta, t)}{\partial r}+\frac{1}{r^{2}} \frac{\partial H(r, \theta, t)}{\partial \theta^{2}}=\sigma^{\prime} \frac{d^{\alpha} B(r, \theta, t)}{d t^{\alpha}}
$$

At node $\mathrm{i}, \mathrm{j}$ :

$\frac{-H\left(r_{i-1}, \theta_{j}, t\right)+2 H\left(r_{i}, \theta_{j}, t\right)-H\left(r_{i+1}, \theta_{j}, t\right)}{(\Delta r)^{2}}+\frac{1}{r} \frac{H\left(r_{i}, \theta_{j}, t\right)-H\left(r_{i-1}, \theta_{j}, t\right)}{\Delta r}+\frac{1}{r^{2}} \frac{-H\left(r, \theta_{j-1}, t\right)+2 H\left(r_{i}, \theta_{j}, t\right)-H\left(r_{i}, \theta_{j+1}, t\right)}{\partial \theta^{2}}=\sigma^{\prime} \frac{d^{\alpha} B(r, \theta, t)}{d t^{\alpha}}$

As no defect was firstly taken into account and for symmetrical reasons, a 1D resolution in the radius direction was enough to simulate the rod-shaped specimen correctly. However, considering that our final objective was to anticipate the influence of local defects on the measured signals, a quarter of the magnetic core cross-section was meshed (see Fig. 6 below). Defects were taken into account afterward through local variations (a few nodes) of the physical properties (magnetic, electrical).
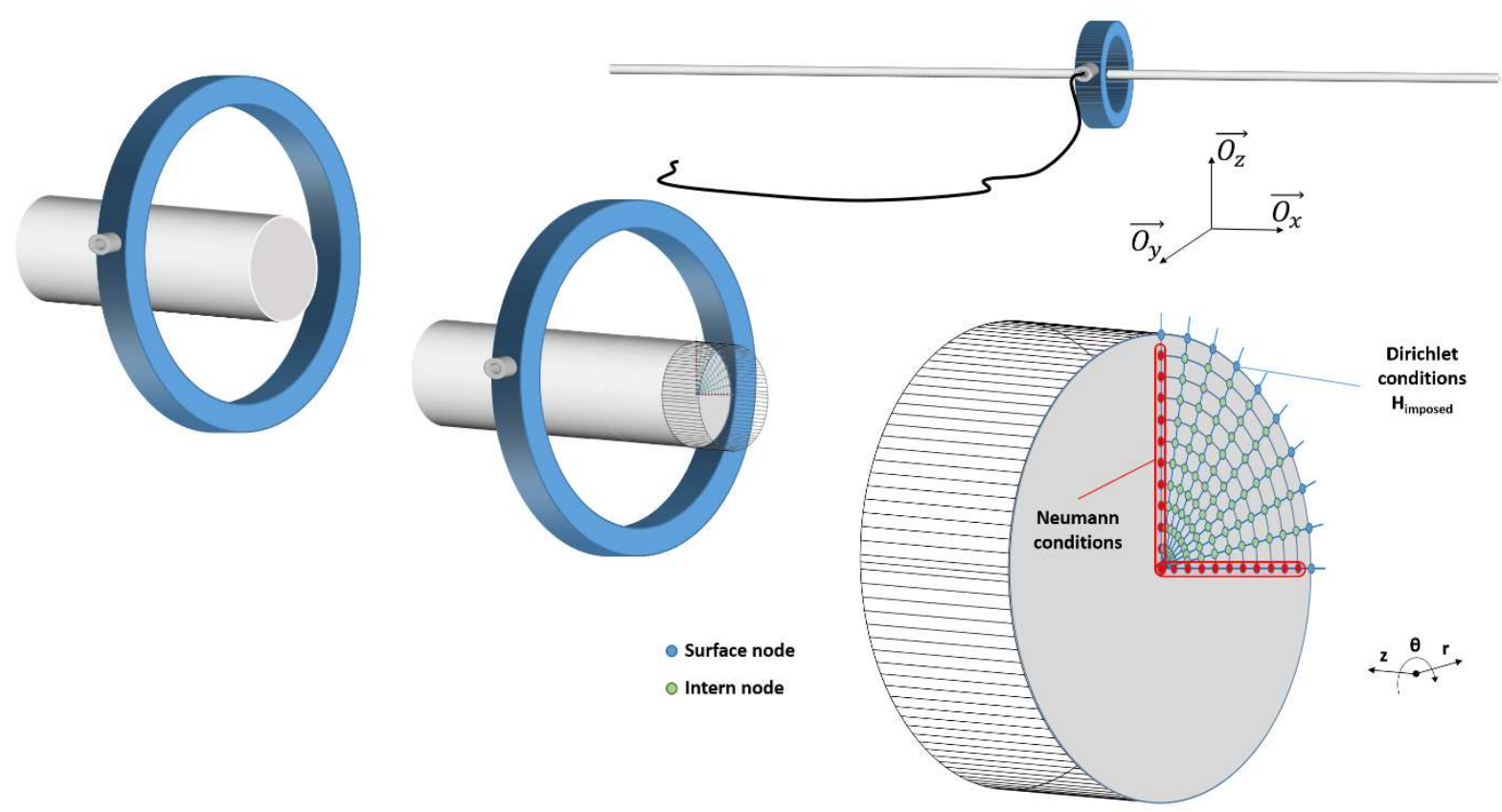

Fig. 6 - Finite differences space discretization, boundary conditions.

Surface excitation field $\mathrm{H}$ was imposed on the blue nodes (Dirichlet boundary conditions, Fig. 6). For comparison purposes, the waveform of this field was derived from the 
experimental measurements. Neumann boundary conditions were set on the red zone nodes

(Fig. 6) to consider the symmetrical behaviors.

\section{b) Time Fractional derivative of the Induction field B}

Anomalous diffusion processes in complex media have been raising interests within the international scientific community. When an anomalous-type diffusion process is observed, the challenge is to understand the underlying mechanism which causes it. Unlike typical diffusion, anomalous diffusion goes through power laws. According to $\alpha$ (the power order), super-diffusion $(\alpha>1)$ or sub-diffusion $(\alpha<1)$ can be detected. Classic equations describing normal diffusion processes are not suitable for characterizing those complex diffusion processes, including diffusion processes in inhomogeneous or heterogeneous medium (porous media). Fractional diffusion equations were introduced to characterize anomalous diffusion phenomena. When a fractional derivative operator is used in the time-dependent term (like in Eq. 1), the diffusion process is characterized by a long-time heavy tail decay.

Fractional calculus is the mathematical analysis sub-division dedicated to non-integer orders, derivatives, and integrals. Standard derivative orders are entire, while fractional derivatives are characterized by real or complex orders. The first-order time derivative of $f(\mathrm{t})$ at a given time $\mathrm{t}$ can be estimated precisely from $f(\mathrm{t}-\mathrm{dt}$ ) (one time step before), meaning that just the close history is considered in the derivative calculus process. Oppositely, when $\alpha$ is non-entire, the numerical derivative estimation needs a large window of $f$ history to reach a reasonable accuracy. From a physical point of view, a link can be established between the history-dependent fractional calculus process and the history-dependent ferromagnetic dynamical hysteresis. At a given time $t$, the magnetic induction field recorded by the eddy current TR probe is obtained by averaging local magnetic states which, according to their 
depth position and the diffusion process, have been excited by the previous state (t-n.dt) of the surface excitation field.

Many definitions exist to describe fractional derivatives. Among all these definition, Liouville [19]-[21] (Eq. 4), Riemann-Liouville [22], Grünwald-Letnikov [23]-[25] and Caputo [26], are the most known:

$$
\frac{d^{\alpha} f(t)}{d t^{\alpha}}=D^{\alpha} f(t)=\frac{1}{\Gamma(1-\alpha)} \frac{d}{d t} \int_{-\infty}^{t}(t-\tau)^{-\alpha} f(t) d \tau, \quad-\infty<t<+\infty
$$

Eq. 4 can be described as the convolution between $f(t)$ and $\operatorname{ta}^{\alpha} H(t) / \Gamma(1-\alpha)$. $\Gamma(\alpha)$ is the gamma function. In the Fourier domain, a first-order derivative is a jw multiplication while a fractional derivative a $(j \omega)^{\alpha}$ multiplication [27]-[29]. For the numerical resolution of Eq. 3, the direct Grünwald-Letnikov derivative definition has been selected:

$$
\begin{gathered}
\frac{d^{\alpha} f(t)}{d t^{\alpha}}=D^{\alpha} f(t)=\lim _{h \rightarrow 0} \frac{1}{h^{\alpha}} \sum_{k=0}^{\infty}(-1)^{k}\left(\begin{array}{l}
\alpha \\
k
\end{array}\right) f(t-k h) \\
\left(\begin{array}{l}
\alpha \\
k
\end{array}\right)=\frac{\alpha(\alpha-1)(\alpha-2) \ldots(\alpha-k+1)}{\alpha !}
\end{gathered}
$$

\section{c) Combined resolution}

By combining Eq. 3 and 5, we obtained the equation to be solved for every node of the space discretized area:

$\frac{-H\left(r_{i-1}, \theta_{j}, t\right)+2 H\left(r_{i}, \theta_{j}, t\right)-H\left(r_{i+1}, \theta_{j}, t\right)}{(\Delta r)^{2}}+\frac{1}{r} \frac{H\left(r_{i}, \theta_{j}, t\right)-H\left(r_{i-1}, \theta_{j}, t\right)}{\Delta r}+\frac{1}{r^{2}} \frac{-H\left(r, \theta_{j-1}, t\right)+2 H\left(r_{i}, \theta_{j}, t\right)-H\left(r_{i}, \theta_{j+1}, t\right)}{\partial \theta^{2}}=\sigma^{\prime} \lim _{h \rightarrow 0} \frac{1}{h^{\alpha}} \sum_{k=0}^{\infty}(-1)^{k}\left(\begin{array}{l}\alpha \\ k\end{array}\right) B\left(r_{i}, \theta_{j}, t-k h\right)$

A matrix system was derived from all these equations:

$$
[M][X]=[S]
$$

Where $\mathrm{M}$ was the stiffness matrix, $\mathrm{X}$ the unknown $H_{i}$ vector, and $\mathrm{S}$ a vector filled from the remaining terms. In all the numerical tests we did, the time discretization was coarse enough to set $k$ equal to the number of previous step time. The matrix system construction, such as the boundary conditions taken into account, needed special attention. $B_{i, j}$ and $H_{i, j}$ were 
connected locally through a nonlinear anhysteretic relation described by a hyperbolic sigmoid function:

$$
B_{i, j}^{a n h}=B_{S} \tan ^{-1}\left(\frac{H_{i, j}}{a}\right)
$$

$B_{i, j}$ was the local induction, $B_{s}$ the saturation induction, and $a$ the anhysteretic induction trajectory parameter. Eq. 3 was not used "as is" in the simulation but through the related permeability, expressed below as a function of $B_{i, j}$ :

$$
\mu_{i}^{a n h}\left(B_{i, j}^{a n}\right)=\frac{B_{S} a}{\left(1+\tan ^{2}\left(\frac{B_{i, j}^{a n h}}{B_{S}}\right)\right)}
$$

The time resolution of Eq. 7 led to the time evolution of all local $B_{i, j}$ and $H_{i, j}$. To compare with the experimental observations, the average induction $B$ was calculated using Eq. 10 below:

$$
B=\frac{\emptyset}{S}=\frac{\sum_{1}^{n} \frac{n_{i, j}}{s_{i, j}}}{S} \text { with } S_{i, j}=r_{i} \cdot d r . d \theta
$$

$\mathrm{n}$ is the number of nodes, $\varnothing$ is the cross-section magnetic flux, and $\mathrm{S}$ the cross-section area. Even if $B_{i, j}$, and $H_{i, j}$ were linked locally through an anhysteretic relation (Eq. 8), the fractional diffusion equation generates losses, and hysteresis behavior was observed when plotting $B$ as a function of $H$ (the tangent surface excitation field). Fig. 7 below depicts the evolution of these average dynamic hysteresis losses through the cross-section of the rod-shaped specimen as a function of $f, \alpha$ and for different values of the electrical conductivity. These results were obtained under imposed amplitude $\left(H_{\max }=1500 \mathrm{~A} \cdot \mathrm{m}^{-1}\right)$ harmonic-type surface magnetic excitation field, $a$ and $B_{s}$ have been set arbitrarily to $0.008 \mathrm{~A} \cdot \mathrm{m}^{-1}$ and $1.5 \mathrm{~T}$, respectively. These dynamic hysteresis losses $<A>$ were calculated through the integration of the resulting hysteresis loop (Eq. 11):

$$
<A>=\int_{0}^{T} B(t) d H(t)
$$


Three sizes of radius were tested. In all these simulation results, both the radius and the angle step were maintained constant.
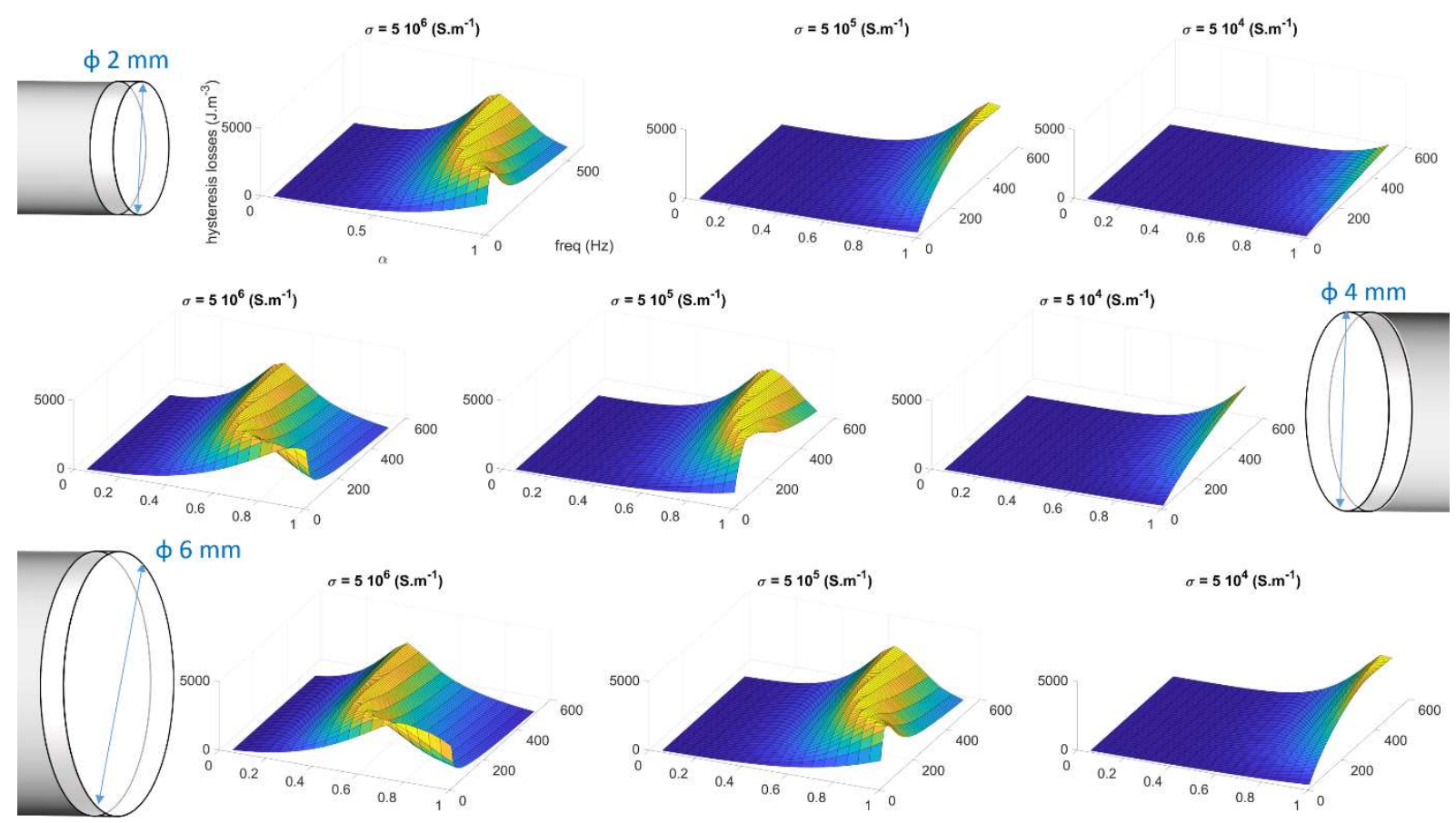

Fig. 7 -Variations of the hysteresis losses as a function of $\alpha$ (the fractional order), $\sigma$ the electrical conductivity, and $\phi$ the cross-section diameter.

A close observation of Fig. 7 leads to the following conclusions:

_ whatever the fractional-order, or the electrical conductivity, the frequency dependence of the hysteresis cycle area always follows the same trajectory: It remains unchanged for low frequency, weak conductivity, and weak fractional derivative orders. Once the quasi-static threshold is reached, it grows up to a maximum value then decreases continuously until a complete disappearance under extreme frequency levels when a shield of macroscopic eddy currents prevents magnetic penetration in the tested specimen. This behavior can be assimilated to a Gaussian curve of increasing average value and standard deviation as $\alpha$ is increasing.

The magnetization distribution through the cross-section of a rod-shaped specimen (1.54 $\mathrm{mm}$ radius) is depicted in Fig. 8 below. Four levels of frequencies were tested. A complete period of magnetic excitation is imposed, and the color scale is set on the maximum value of 
the resulting local induction field $B_{i}$. As predicted, the magnetization is homogeneous under low-frequency levels. Oppositely, for large frequencies, the skin effect (rejection of the Eddy currents over the cross-sectional peripheral areas, reduction of the magnetized area) is evident. These first comparisons to experimental results allow concluding too over the good accuracy of the simulation method. The simulation parameters have been set to $\sigma^{\prime}=510^{6}, \alpha$ $=0.61, \mathrm{a}=0.0078$ and $\mathrm{Bs}=1.89 \mathrm{~T}$ using the minimization of an error function: the mean relative standard deviation (Eq. 12 below).

$$
\operatorname{Err}(\%)=\frac{100}{q} \sum_{i=1}^{q} \frac{\left|W_{\text {meas }_{i}}-W_{\text {sim }_{i}}\right|}{W_{\text {meas }_{i}}}
$$

(a and Bs do not influence the hysteresis losses frequency dependence. Even if they are different from those of the parametric study, Fig. 7 results are still valid with this new set of parameters).

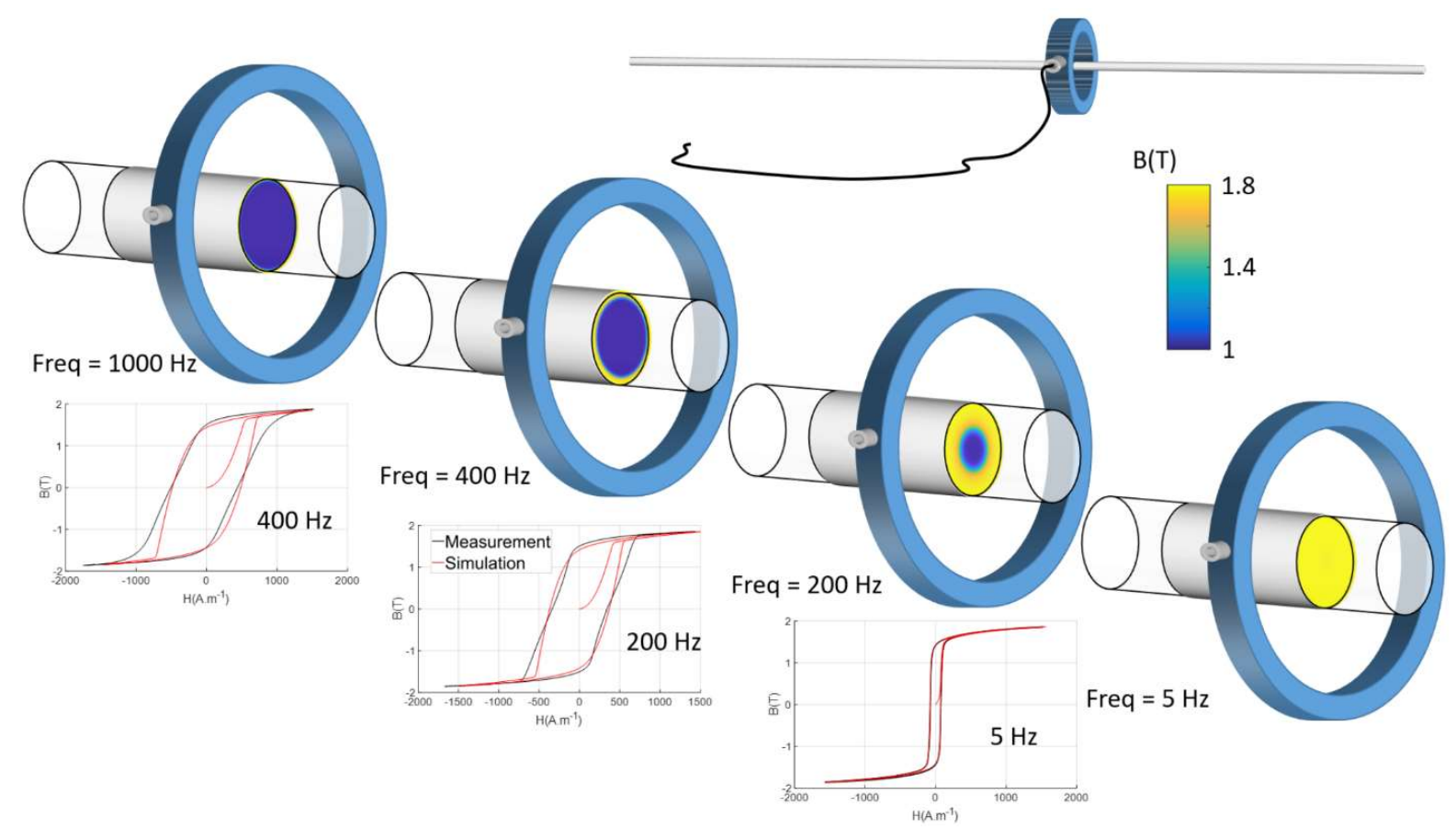

Fig. 8 - Magnetic state distribution through the cross-section of a simulated specimen, comparison measurement/Simulation for three frequency levels.

The good comparative results between the simulated and the measured hysteresis cycles for three levels of frequencies depicted in Fig. 8 gave us a first good confirmation of the 
simulation method potential. However, a slight deviation of the simulated cycles can be observed close to the saturation elbow when the frequency is high. Multiple reasons can be found to justify this observation, including a wrong consideration of the magnetization rotation by the simulation process.

\section{d) Defect consideration}

Due to high-temperature variations combined with intense mechanical stress treatments during the manufacturing process, the steel rod-shaped specimens tested in this study exhibit unperfect surface state and sometimes macroscopic defects limiting their quality and reducing their expected lifetimes. Early-stage inspection is mandatory to extract uncertain specimens from the manufacturing production lines. The type of defects expected is macroscopic surface cracks or macroscopic structural modification visible to the naked eye. In the simulation, these surface defects were taken into account over changes in the cross-section geometry (Fig. 9) or local variations of the physical properties (electrical conductivity, magnetic behavior).
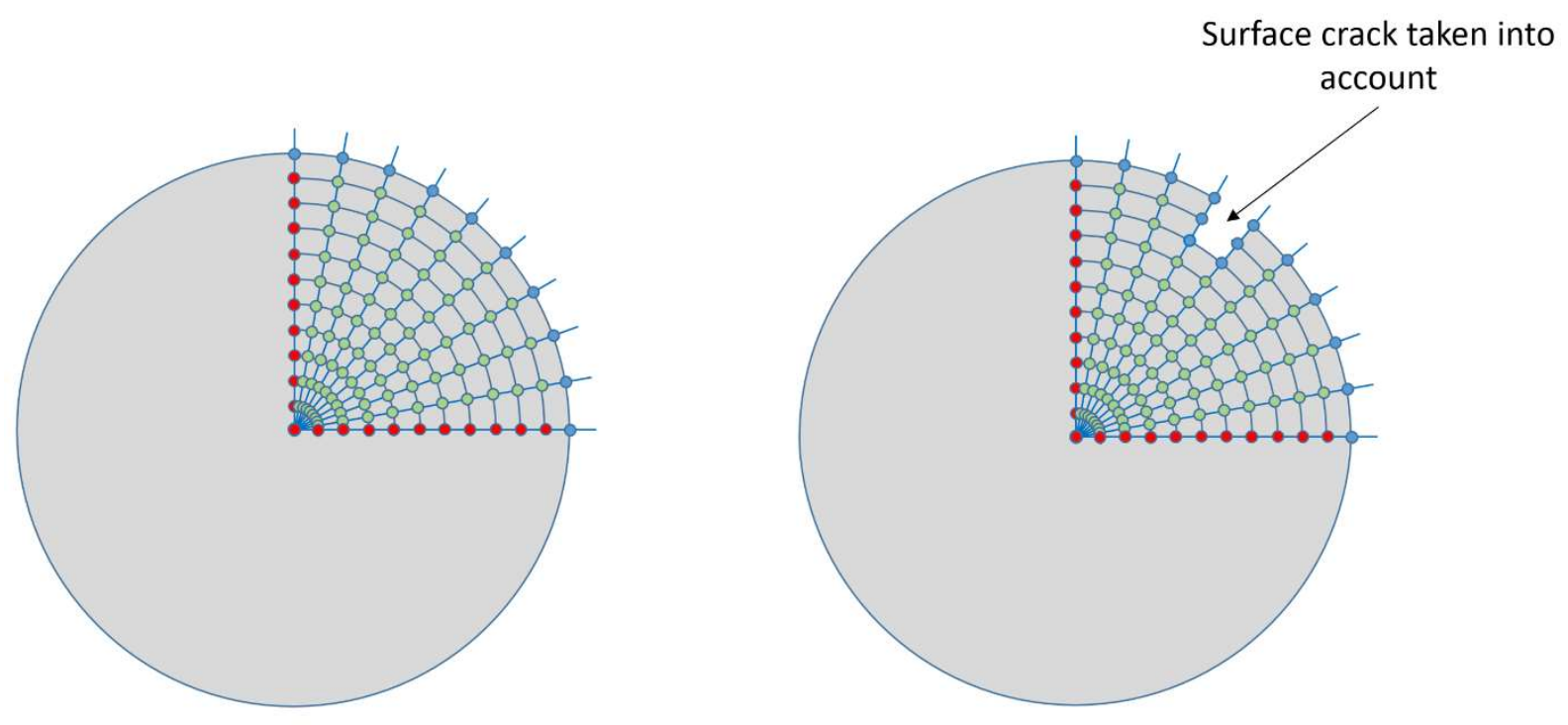

Fig. 9 - Local modification of the geometrical cross-section for the consideration of a surface macroscopic defect.

The defect presence is distorting the magnetic state distribution, as depicted in Fig. 10. In this series of tests, the frequencies and the simulation parameters were set similar to those 
of Fig. 8. The distribution was especially high at $400 \mathrm{~Hz}$ when the defect depth was close to the skin depth $\left(\delta \approx 350 \mu \mathrm{m}\right.$, with $\mu_{\mathrm{r}}=1000$ (set close to the middle of the differential permeability extrema to establish a quantitative estimation) and $\sigma^{\prime}=510^{6}$ ).
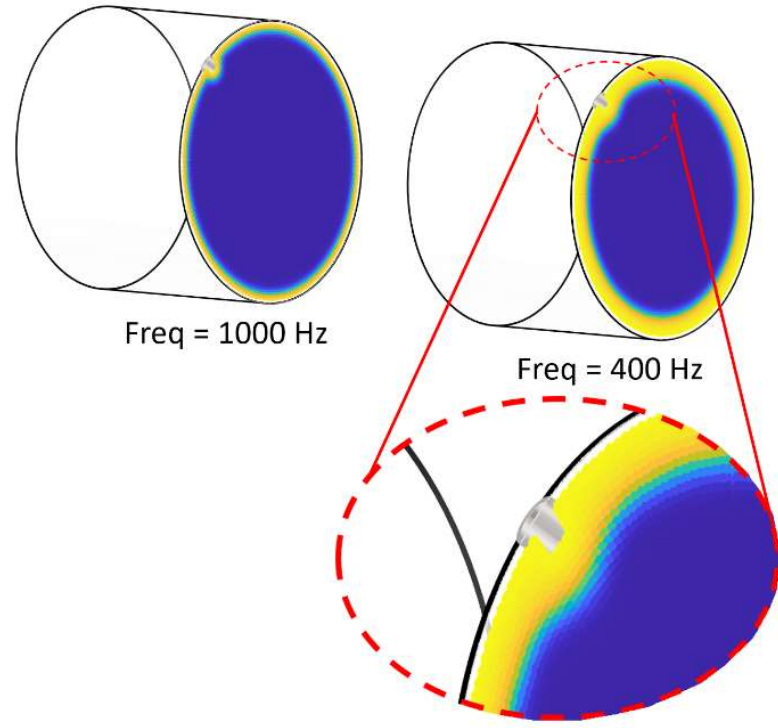

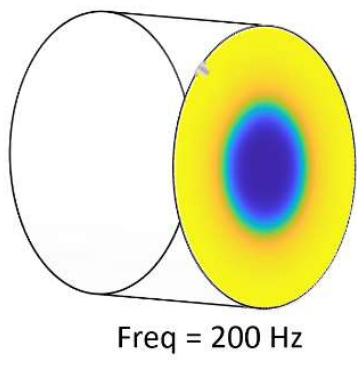

$B(T)$

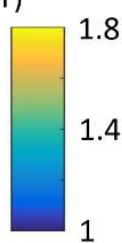

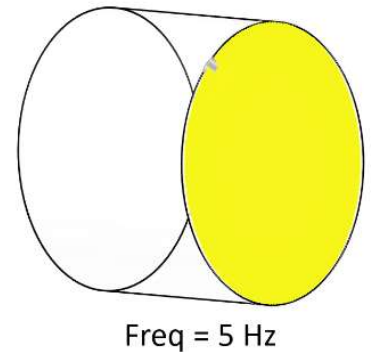

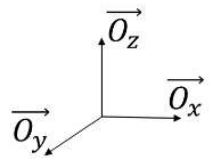

Fig. 10 - Magnetic state distribution, including an additional defect (hole of $500 \mu \mathrm{m}$ diameter, $300 \mu \mathrm{m}$ depth).

Distortions of the magnetic lines due to the defect presence are not considered. $\vec{H}$ and $\vec{B}$ are still supposed to have only non-zero components along the z-axis. The real-life situation is probably slightly different, and improvement could be envisaged by switching to a 3D simulation, the adjunction of an airbox, and a vector consideration of the magnetic quantities.

\section{4 - Experimental validation}

The tested specimens, including the additional defect, were translating in the horizontal direction at a speed of $8 \mathrm{~mm} \cdot \mathrm{s}^{-1}$. During its movement, the flaw was successively crossing the first and the second flux sensor coil creating a sudden variation in the monitored differential voltage (see Fig. 11 below). The speed of $8 \mathrm{~mm} / \mathrm{s}$ is low. It is, however, well adapted to the weak magnetic excitation frequency $(500 \mathrm{~Hz})$. Faster speed can be used when working for surface flaws detection and when necessary, like in an industrial environment. 


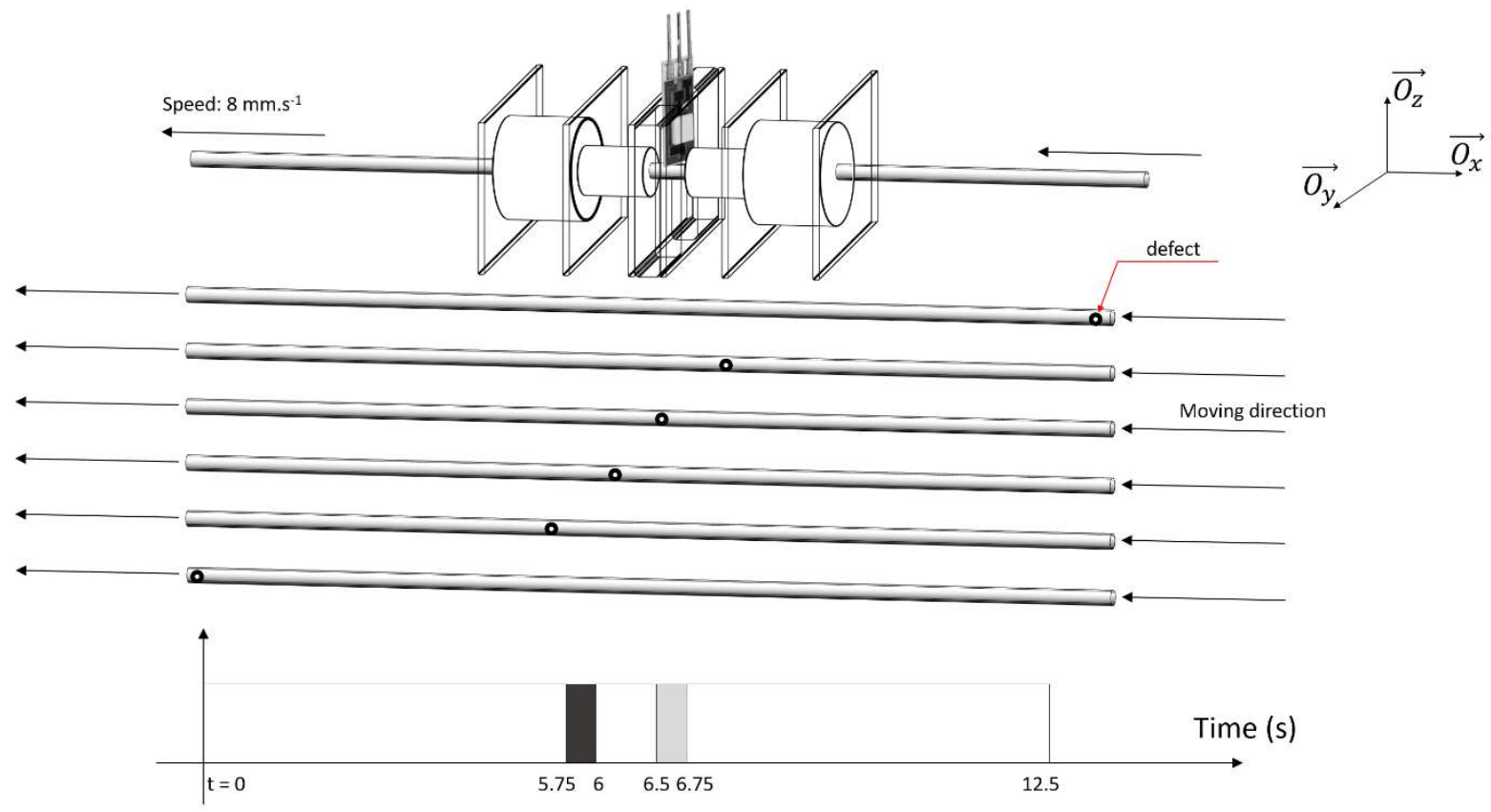

Defect in the first coil scanned area

No defect in the scanned areas

Defect in the second coil scanned area

Fig. 11 - Illustration of the tested specimen translation; timeline of the defect movement.

From the simulation point of view, the electromotive force measured by each sensor coil was obtained through the time derivation of the average magnetic induction:

$$
e=n \cdot S \cdot \frac{d B}{d t}
$$

It was obtained by subtracting the electromotive force of the second sensor from the first one. The average magnetic induction was calculated from a homogeneous cross-section answer during the "no defect" period of time and from the modified one meanwhile. The simulation parameters were those of Fig. 8. $d r$ and $d \vartheta$ were set to $0.0049 \mathrm{~mm}$ and $0.0132 \mathrm{rd}$ respectively, for a total of 57117 nodes. These values gave the best compromise between accuracy and simulation time. The modification of the cross-section was realized according to the defect description provided in section 3.c. The RMS value of the resulting signals was calculated to be compared to the experimental measurements. The timeline of the differential ECT simulated electromotive forces between the first and the second coil is displayed in the three top chronograms of Fig. 11 below. Finally, a comparison between the RMS value of the 
measured and the simulated differential electromotive forces is visible in the last two chronograms of Fig. 12.

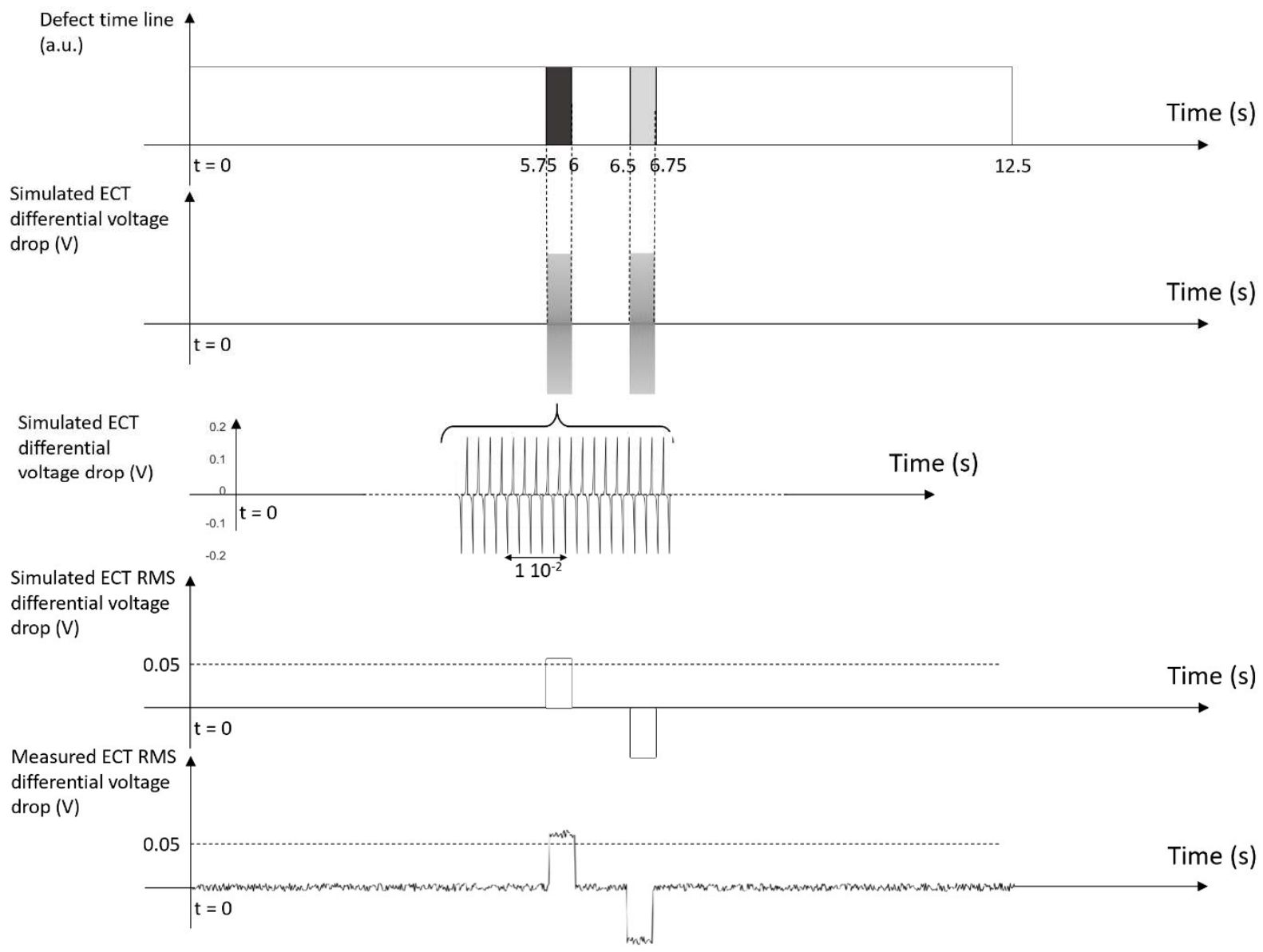

Fig. 12 - Time dependence of the simulated ECT differential voltage drop, comparison between the simulated and the measured RMS differential voltage drop.

The mean relative standard deviation (Eq. 12) has been used to quantitively check the accuracy of the simulation method. This deviation has been calculated using all the space discretization separating $t=5.5 \mathrm{~s}$ and $t=7 \mathrm{~s}$ (Fig. 12). A low $3.4 \%$ relative error has been obtained. Such good accuracy leads to the validation of the simulation method.

\section{5 - Conclusion:}

The magnetic behaviour of a ferromagnetic rod-shaped steel specimen is sensitive to multiple parameters, including the temperature, the mechanical state, the microstructural content ... Under stable conditions, ECT can be used as an indirect way to identify and 
characterize one of these parameters. ECT is the concept of using the material electromagnetic signature to anticipate a level of integrity. ECT is widely used already, but simulation tools able to improve the performances and interpretation of the resulting signals are still highly expected. In this manuscript, a simulation method based on the resolution of a fractional magnetic field diffusion equation has been proposed to simulate the ECT answer of an ECT sensor, i.e. two wounded coils in a differential configuration and under the presence of a surface flaw. We opted for the ECT differential method because it is popular in the industrial environment, especially for this type of geometry. The numerical method, which consists of the simulation of both the sensor coils and returns their differential answer, can easily be adapted to a single-coil situation.

The diffusion of the magnetic field in the ferromagnetic rod-shaped steel specimen is distorted by the domain wall kinetic. The interaction between the domains and the macroscopic eddy currents generate additional lag times and modify the diffusion process, which can be classified as anomalous. By converting the time derivative term of the classic diffusion equation into a fractional derivative one and by adjusting the fractional order of this new diffusion equation, the additional lag can be correctly taken into account.

This simulation method cannot be seen as an alternative to the classical eddy current but must be considered as an elegant way to take into account the nonlinearity and frequency dependence of hysteresis. Even if solved combined to finite differences, finite elements could be used as well.

Many suggestions can be made as perspectives of this work:

_ The discretization strategy has been set in a first choice to the rod cross-section but considering the relative uniformity of the defect geometry, the $(r, z)$ plan or a 3D discretization 
would have made sense too. Both these options are worth the investigation and will be subjected to further investigation.

_ Different natures and geometries of flaws should be tested and simulated. We can clearly envisage using our simulation tool as a way to anticipate signals and identify defects.

_ As a large proportion of ECT devices use pancake coils for the scan of plan surfaces, one of the following steps of this work will be dedicated to the simulation of such experimental situations. Here again, time-fractional derivative operators will be proposed for the time dynamic of the diffusion process.

Acknowledgments: This project is supported by the Program of National Natural Science Foundation of China (Grant No. 51805298), Natural Science Foundation of Shandong Province (ZR201807090390), Young Scholars Program of Shandong University, Weihai (Grant No. 20820201004), and Fundamental Research Funds for the Central Universities (2019ZRJC006) 


\section{References}

[1] J. Garcia-Martin, J. Gomez-Gil, E. Vasquez-Sanchez, "Nondestructive techniques based on Eddy current testing", Sensors, vol. 11, iss. 3, pp. 2525-2565, 2011.

[2] A. Sophian, G. Tian, M. Fan, "Pulsed Eddy current Nondestructive Testing and Evaluation: A review", Chin. J. of Mech. Eng., vol. 30, pp. 500-514, 2017.

[3] B.A. Auld, J.C. Moulder, "Review of advances in quantitative Eddy current nondestructive evaluation", J. of Nondest. Eval., vol. 18, pp. 3-36, 1999.

[4] B. Helifa, A. Oulhadj, A. Benbelghit, I.K. Lefkaier, F. Boubenider, D. Boutassouna, "Detection and measurement of surface cracks in ferromagnetic materials using eddy current testing", NDT\&E Int., vol. 39, iss. 5, pp. 384-390, 2006.

[5] A. Merahi, K. Medles, M. Bardadi Bendaha, A. Tilmartine, "Design and development of a low cost technique for sorting household wastes using eddy current separation process", Int. J. of Env. Stud., vol. 73, iss. 2, 2016.

[6] Z. Xu, X. Wu, J. Li, Y. Kang, "Assessment of wall thinning in insulated ferromagnetic pipes using the time-to-peak of differential pulsed eddy-current testing signal", NDT\&E Int., vol. 51, pp. 24-29, 2012.

[7] T. Takagi, M. Hashimoto, H. Fukutomi, M. Kurokawa, K. Miya, H. Tsuboi, M. Tanaka, J. Tani, T. Serizawa, Y. Harada, E. Okano, R. Muarakami, "Benchmark models of eddy current testing for steam generator tube: Experiment and numerical analysis", Int. J. of App. Electromag. in Mat., vol. 5, iss. 3, pp. 149-162, 1994.

[8] N. Bowler, Y. Huang, "Electrical conductivity measurement of metal plates using broadband eddycurrent and four-point methods", Meas. Sci. and Tech., vol. 16, n¹1, 2005.

[9] J. Cheng, J. Qiu, X. Xu, H. Ji, T. Takagi, T. Uchimoto, "Research advances in eddy current testing for maintenance of carbon fiber reinforced plastic composites", Int. J. of App. Electr. and Mech., vol. 51, n³, pp. 261-284, 2016.

[10] F. Kojima, T. Takagi, T. Matsui, "Inverse methodology for Eddy current testing using transmitterreceiver coil probes", AIP Conf. Proc. 700, 643, 2004.

[11] A. Rosell, "Efficient finite element modelling of eddy current probability of detection with transmitter-receiver sensors", NDT\&E Int., vol. 75, pp. 48-56, 2015.

[12] T. Chady, M. Enokizono, "Crack detection and recognition using an eddy current differential probe", IEEE Trans. Magn. vol. 35, n³, pp. 1849-1952, 2020.

[13] J.P.A. Bastos, N. Sadowski, "Magnetic materials and 3D finite element modeling", CRC Press, Boca Raton, 2014.

[14] B. Gupta, B. Ducharne, G. Sebald, T. Uchimoto, "A space discretized ferromagnetic model for nondestructive eddy current evaluation", IEEE Trans. Magn., vol. 54 Iss. 3, 2018.

[15] C. Guérin, K. Jacques, R.V. Sabariego, P. Dular, C. Geuzaine, J. Gyselinck, "Using a Jiles-Atherton vector hysteresis model for isotropic magnetic materials with the finite element method, Newton- 
Raphson method, and relaxation procedure", Int. J. of Num. Mod.: Electr. Net., Dev. and Fie., vol. 30, iss. 5, 2016.

[16] M. A. Raulet, B. Ducharne, J.P. Masson, and G. Bayada, "The magnetic field diffusion equation including dynamic hysteresis: a linear formulation of the problem", IEEE Trans. Magn., vol. 40, $n^{\circ} 2$, pp. $872-875,2004$.

[17] B. Ducharne, Y.A. Tene Deffo, B. Zhang, G. Sebald, "Anomalous fractional diffusion equation for magnetic losses in a ferromagnetic lamination", The Eur. Phys. J. Plus, 135:325, 2020.

[18] B. Ducharne, Y.A. Tene Deffo, P. Tsafack, B. Zhang, G. Sebald, "Anomalous fractional magnetic field diffusion through the cross-section of massive toroidal ferromagnetic core", Com. in Nonlin. Sci. and Num. Sim., vol. 92, 105450, 2021.

[19] K. S. Miller and B. Ross, "An Introduction to the Fractional Calculus and Fractional Differential Equations", John Wiley \& Sons, New York, NY, USA, 1993.

[20] K. B. Oldham and J. Spanier, "The Fractional Calculus: Theory and Application of Differentiation and Integration to Arbitrary Order", Academic Press, New York, NY, USA, 1974.

[21] J. T. Machado, V. Kiryakova, and F. Mainardi, "Recent history of fractional calculus", Communications in Nonlinear Science and Numerical Simulation, vol. 16, n 3, pp. 1140-1153, 2011.

[22] B. Riemann, "Versuch Einer Allgemeinen Auffassung der Integration und Differentiation", Gesammelte Mathematische Werke und Wissenschaftlicher Nachlass, Teubner, Leipzig, 1876, Dover, New York, NY, USA, 1953.

[23] A. K. Grünwald, "Über "begrenzte" derivationen und deren anwendung", Zeitschrift für Mathematik und Physik, vol. 12, pp. 441-480, 1867.

[24] A. V. Letnikov, "Theory of differentiation with an arbitrary index", Sbornik: Mathematics, vol. 3, pp. 1-66, 1868 (Russian).

[25] B. Ducharne, G. Sebald, D. Guyomar, G. Litak, "Fractional model of magnetic field penetration into a toroidal soft ferromagnetic sample", Int. J. of Dyn. And Cont., pp. 1-8, 2017.

[26] M. Caputo, "Linear models of dissipation whose $q$ is almost frequency independent-ii", Geophysical Journal of the Royal Astronomical Society, vol. 13, no. 5, pp. 529-539, 1967.

[27] B. Zhang, B. Gupta, B. Ducharne, G. Sebald, T. Uchimoto, "Dynamic magnetic scalar hysteresis lump model, based on JilesAtherton quasi-static hysteresis model extended with dynamic fractional derivative contribution", IEEE Trans. on Mag., iss. 99, pp. 1-5, 2018.

[28] B. Zhang, B. Gupta, B. Ducharne, G. Sebald, T. Uchimoto, "Preisach's model extended with dynamic fractional derivation contribution", IEEE Trans. on Mag., vol. 54, iss. 3, 2017.

[29] B. Ducharne, B. Newell, G. Sebald, "A unique fractional derivative operator to simulate all dynamic piezo ceramic dielectric manifestations: from aging to frequency dependent hysteresis", IEEE Transactions on Ultrasonic, Ferroelectricity and Frequency Control, vol. 67, iss. 1, pp. 197-206, 2019. 